\title{
Symmetry dependence of phonon lineshapes in superconductors with anisotropic gaps
}

\author{
T. P. Devereaux \\ Department of Physics \\ University of California \\ Davis, CA 95616
}

\begin{abstract}
The temperature dependence below $T_{c}$ of the lineshape of optical phonons of different symmetry as seen in Raman scattering is investigated for superconductors with anisotropic energy gaps. It is shown that the symmetry of the electron-phonon vertex produces non-trivial couplings to an anisotropic energy gap which leads to unique changes in the phonon lineshape for phonons of different symmetry. The phonon lineshape is calculated in detail for $B_{1 g}$ and $A_{1 g}$ phonons in a superconductor with $d_{x^{2}-y^{2}}$ pairing symmetry. The role of satellites peaks generated by the electronphonon coupling are also addressed. The theory accounts for the substantial phonon narrowing of the $B_{1 g}$ phonon, while narrowing of the $A_{1 g}$ phonon which is indistinguishable from the normal state is shown, in agreement with recent measurements on $\mathrm{Bi}_{2} \mathrm{Sr}_{2} \mathrm{CaCu}_{2} \mathrm{O}_{8}$ and $\mathrm{YBa}_{2} \mathrm{Cu}_{3} \mathrm{O}_{7}$.
\end{abstract}

PACS numbers: 74.20.Fg, 74.30.Gn, 74.60.-w, 74.65.+n

Typeset Using REVTEX 


\section{INTRODUCTION}

Optical phonons observed via Raman scattering have provided a large amount of information concerning the energy gap in high- $\mathrm{T}_{c}$ superconductors [1], and there have been attempts to describe the changes in the phonon lineshapes below $\mathrm{T}_{c}$ in $s$-wave [2] and $d$-wave superconductors [3]. It is believed that the changes in the phonon lineshape below $\mathrm{T}_{c}$ are due in part to changes in the phonon self-energy resulting from coupling between phonons and quasiparticles. It has been argued that if the optical phonon has a frequency below the pair threshold energy $2 \Delta$, then the phonon's linewidth decreases (narrows) and its frequency renormalizes to lower frequencies (softens) as the quasiparticles become frozen out. However, for a phonon near $2 \Delta$, the linewidth is predicted to grow due to the enhancement of the density of states at the gap edge and there can be either pronounced phonon softening or hardening depending on which side of the threshold the phonon is located. This simple picture has been employed to determine the position of $2 \Delta$ in the cuprate superconductors.

However, this simple analysis applied to the cuprate systems has revealed that the above picture is a bit misleading. The above scenario has yielded a value for the energy gap that is different for different types of optical phonons and is thus symmetry-dependent. For the case of the Bi 2:2:1:2 system, where very clean surfaces can be obtained, a low frequency phonon which transforms according to $A_{1 g}$ symmetry (located at $464 \mathrm{~cm}^{-1}$, connected with the bridging Oxygen vibrations) shows a downward frequency shift (softening) while no substantial linewidth change from the normal state can be resolved from the data [4]. However, the $B_{1 g}$ phonon $\left(285 \mathrm{~cm}^{-1}\right.$, connected with the antisymmetric out of plane $\mathrm{O}(2)$ and $\mathrm{O}(3)$ vibrations in the $\mathrm{Cu}-\mathrm{O}$ plane) on the contrary shows a small frequency softening but a substantial linewidth narrowing below $\mathrm{T}_{c}$ [5]. Similar behavior is seen in the YBCO systems [6], where such a large difference in behavior between the $A_{1 g}$ and $B_{1 g}$ phonons in part led the authors of Ref. [6] to suggest that these two phonons interact with different electronic systems.

There has been no satisfactory theoretical explanation for the behavior of the different phonons. The main problem in addressing these experiments with the existing theories concerns the lack of attention paid to the symmetry dependence of the optical phonons. However, this symmetry dependence can be an important tool to uniquely determine the

$\hat{\mathbf{k}}$-dependence of the energy gap around the Fermi surface. It has been shown that the electronic contribution to Raman scattering can provide a large amount of polarization (symmetry) dependent information that allows for a stringent test to made to determine the 
actual symmetry of the energy gap in superconductors [7]. It was shown that the coupling between the Raman vertex and an anisotropic gap leads to symmetry dependent spectra, with peak positions and low frequency and temperature behavior dependent on polarization orientations. These changes in the spectra allow for a direct determination of $|\Delta(\hat{\mathbf{k}})|$. Good agreement with the electronic Raman spectra taken on very clean BSCCO surfaces was obtained using a gap which was predominantly or entirely of $d_{x^{2}-y^{2}}$ symmetry, where the peak position and the low frequency behavior of the spectra could be straightforwardly accounted for. The symmetry dependence of the data led to the conclusion that the gap must be predominantly of $B_{1 g}$ character. Since the phonon self-energy is very similar to the electronic Raman density response, the same type of analysis for the electronic contribution to Raman scattering can be made to the phonons as well, leading to a further check on the predictions recently made concerning the energy gap in the cuprate materials.

We propose an alternative explanation for the symmetry dependence of the Raman shifts based upon nontrivial couplings of phonons of different symmetry with an anisotropic energy gap. Close attention will be paid to the role of the electron-phonon vertex, and consequences of its k-dependence will be addressed. Most importantly, it is shown that the lineshape is polarization dependent for anisotropic superconductors and different dependences on temperature can be used to determine not only the magnitude but the symmetry dependence of the energy gap. Moreover, it is shown that the peak of the self energy can be located at frequencies below $2 \Delta_{\max }$ for certain polarizations which have an symmetry orthogonal to that of the energy gap. Thus if the symmetry of the phonon is neglected, values of the energy gap inferred from changes in the phonon lineshape using an isotropic s-wave theory will be underestimated. In particular, the phonon spectral function for a superconductor with $d_{x^{2}-y^{2}}$ symmetry is examined and a comparison is made with experimental data on both the $B_{1 g}$ and the $A_{1 g}$ phonons in BSCCO and YBCO. It is shown that satisfactory agreement can be obtained which reconciles the differences between the $A_{1 g}$ and $B_{1 g}$ phonon lineshapes.

\section{PHONON SPECTRAL FUNCTION}

The phonon spectral function is given by

$$
\operatorname{Im} D(\omega)=\frac{4 \omega_{0}^{2} \Sigma^{\prime \prime}(\omega)}{\left[\omega^{2}-\omega_{0}^{2}-2 \omega_{0} \Sigma^{\prime}(\omega)\right]^{2}+4 \omega_{0}^{2} \Sigma^{\prime \prime 2}}
$$


where $\omega_{0}$ is the optical phonon frequency and $\Sigma^{\prime}, \Sigma^{\prime \prime}$ are the real and imaginary parts of the phonon self-energy, respectively. The real part of the self energy renormalizes the position of the phonon, while the imaginary part governs the linewidth. The interaction of optical phonons and electrons can be simply written as

$$
H_{e-p h}=\sum_{\mathbf{k}, \mathbf{q}, \gamma, \sigma} g_{\mathbf{k}}^{\gamma}(\mathbf{q}) c_{\mathbf{k}-\mathbf{q}, \sigma}^{\dagger} c_{\mathbf{k}, \sigma}\left(b_{\mathbf{q}, \gamma}^{\dagger}+b_{\mathbf{q}, \gamma}\right),
$$

where $g_{\mathbf{k}}^{\gamma}(\mathbf{q})$ is the matrix element for scattering an electron from $\mathbf{k} \rightarrow \mathbf{k}-\mathbf{q}$, and $b_{\mathbf{q}, \gamma}, b_{\mathbf{q}, \gamma}^{\dagger}$ are the field operators for phonons of branch $\gamma$. The details of the scattering matrix elements depend on the nature of the mechanism of the electron-phonon coupling and the symmetry of the lattice vibration. In this paper we only consider the symmetry of the matrix element and leave a treament of the mechanism and magnitude of the coupling for future consideration [8].

We take the $\mathbf{k}$ dependence along the Fermi surface of the vertex into account by expanding in terms of Fermi surface harmonics $\Phi$ for small $\mathbf{q}$,

$$
g_{\mathbf{k}}^{\gamma}=\sum_{L} g_{L}^{\gamma} \Phi_{L}^{\gamma}(\hat{\mathbf{k}})
$$

where the index $L$ indicates the order of polynomial that transforms according to the $\gamma-t h$ representation of the point group of the crystal. For cylindrical Fermi surfaces, $L$ can be replaced by azimuthal quantum numbers. The symmetry of the optical phonon enters into the matrix elements $g_{\mathbf{k}}^{\gamma}$. The matrix elements for the phonons accessible to in-plane polarizations are given for a cylindrical (2-D) Fermi surface in terms of azimuthal angle $\phi$ as

$$
\begin{aligned}
g_{\hat{\mathbf{k}}}^{A_{1 g}} & =g_{L=0}^{A_{1 g}}+g_{L=4}^{A_{1 g}} \sqrt{2} \cos (4 \phi)+\ldots \\
g_{\hat{\mathbf{k}}}^{B_{1 g}} & =g_{L=2}^{B_{1 g}} \sqrt{2} \cos (2 \phi)+\ldots
\end{aligned}
$$

where we have dropped higher order terms, arguing that they are more anisotropic than the terms considering here and will hence be of minor importance. The $L=2$ term for the $A_{1 g}$ channel which is present for $\mathrm{z}$ dispersion is absent here and the $L=4$ term is the first anisotropic term in the series in this case [7]. Also, since there is no dispersion in the $\mathrm{z}$ direction in this case, there are no contributions to the $E_{g}$ channels. Consequences of the Fermi surface and the resulting response functions are considered in a forthcoming publication [9], and thus for our purposes we will confine our attention to only cylindrical Fermi surfaces. 
The form of the $e-p h$ interaction Eq. (2) is similar to the electronic contribution to Raman scattering in the case of non-resonant scattering with the replacement of the effective Raman vertex by the $e-p h$ coupling vertex. Thus we can proceed along the lines recently taken for the case of the electronic Raman scattering [7], where it was shown that the Raman response is extremely polarization dependent for superconductors with an anisotropic energy gap. Moreover, it was shown that the collective modes which appear in the case of $d$-wave superconductors are of little importance to the Raman response [7,9]. We can then separate the self energy in two parts

$$
\Sigma(\mathbf{q}, \omega)=\Sigma(\mathbf{q}=0, \omega)+\delta \Sigma(\mathbf{q}, \omega),
$$

Delaying a discussion of $\delta \Sigma$ until Section III, we can write down the spectrum of the self energy at $q=0$ in the pair approximation, e.g., neglecting collective modes as

$$
\Sigma_{g, g}^{\prime \prime}(\mathbf{q}=\mathbf{0}, \omega)=-\frac{4 N_{F}}{\omega}\left\langle\frac{\left|g_{\hat{\mathbf{k}}}^{\gamma}\right|^{2}|\Delta(\hat{\mathbf{k}})|^{2} \Theta\left(\omega^{2}-4|\Delta(\hat{\mathbf{k}})|^{2}\right)}{\sqrt{\omega^{2}-4|\Delta(\hat{\mathbf{k}})|^{2}}}\right\rangle \tanh (\omega / 4 T) .
$$

The subscript $g, g$ denotes the pair susceptibility calculated with vertices $g$. The real part can be obtained via a Kramers-Kronig transformation. Here $\langle\ldots\rangle$ denotes an average over the Fermi surface, $N_{F}$ is the density of states per spin at the Fermi level, $\Theta$ is a Theta function and $\Delta(\hat{\mathbf{k}})$ is the generalized $\mathrm{k}$-dependent energy gap. We see that if the gap is isotropic, $(\Delta(\hat{\mathbf{k}})=\Delta)$, the average around the Fermi surface is frequency independent and thus the symmetry of the vertex only determines an overall prefactor of the self energy. Also, since the imaginary part of the self-energy has a divergence at the pair threshold energy $2 \Delta$ a phonon with a frequency below the threshold should be infinitely sharp (neglecting strong-coupling effects). However, if the gap is anisotropic, the vertex and gap couple when averaging over the Fermi surface to produce non-trivial changes in the self-energy of phonons of different symmetries. Further, if the gap vanishes on the Fermi surface, the presence of the nodes can provide decay channels for the phonon leading to a finite linewidth for all non-zero frequencies [3].

The isotropic $(L=0)$ density-like terms will be coupled to the long range Coulomb forces and thus we must take screening of the vertex into account. Summing R.P.A. diagrams we recover the known result at $q=0$,

$$
\Sigma^{s c .}=\Sigma_{g, g}-\Sigma_{g, 1}^{2} / \Sigma_{1,1},
$$

where 1 denotes the $L=0$ contribution of the vertex $g$ [10,[1]. Therefore we see that the $L=0$ terms are completely screened for $\mathbf{q}=0$ as a consequence of the long range Coulomb interactions and do not contribute to the Raman response. 
Carrying out the integrations in Eq. (6) using a $d_{x^{2}-y^{2}}$ gap, $\Delta(\hat{\mathbf{k}}, T)=\Delta_{0}(T) \cos (2 \phi)$ for a cylindrical Fermi surface, the spectrum of the phonon self energies can be written down in terms of complete elliptical integrals $K$ and $E$ of the first and second kinds, respectively. Taking screening into account and defining $x=\omega / 2 \Delta_{0}$, we obtain for $T=0$,

$$
\Sigma_{B_{1 g}}^{\prime \prime s c .}=\Sigma_{B_{1 g}}^{\prime \prime}(\mathbf{q}=0, \omega)=\frac{-4 N_{F} g_{B_{1 g}}^{2}}{3 \pi x} \begin{cases}{\left[\left(2+x^{2}\right) K(x)-2\left(1+x^{2}\right) E(x)\right],} & x \leq 1, \\ x\left[\left(1+2 x^{2}\right) K(1 / x)-2\left(1+x^{2}\right) E(1 / x)\right], & x>1,\end{cases}
$$

i.e., the $B_{1 g}$ channel is not affected by Coulomb screening, while

$$
\Sigma_{A_{1 g}}^{s c .}=\Sigma_{A_{1 g}, A_{1 g}}-\Sigma_{A_{1 g}, 1}^{2} / \Sigma_{1,1}
$$

with the spectral functions

$$
\begin{gathered}
\Sigma_{A_{1 g}, A_{1 g}}^{\prime \prime}(\mathbf{q}=0, \omega)=\frac{-4 N_{F} g_{A_{1 g}}^{2}}{15 \pi x} \\
\times \begin{cases}{\left[\left(7-8 x^{2}+16 x^{4}\right) K(x)-\left(7-12 x^{2}+32 x^{4}\right) E(x)\right],} & x \leq 1, \\
x^{4}\left[\left(32-28 / x^{2}+11 / x^{4}\right) K(1 / x)-\left(32-12 / x^{2}+7 / x^{4}\right) E(1 / x)\right], & x>1,\end{cases} \\
\Sigma_{A_{1 g}, 1}^{\prime \prime}(\mathbf{q}=0, \omega)=\frac{-2 \sqrt{2} N_{F} g_{A_{1 g}}}{3 \pi x} \begin{cases}{\left[\left(1+2 x^{2}\right) K(x)-\left(1+4 x^{2}\right) E(x)\right],} & x \leq 1, \\
(1 / x)\left[\left(4-1 / x^{2}\right) K(1 / x)-\left(4+1 / x^{2}\right) E(1 / x)\right], & x>1,\end{cases}
\end{gathered}
$$

and

$$
\Sigma_{1,1}^{\prime \prime}(\mathbf{q}=0, \omega)=\frac{-2 N_{F}}{\pi x} \begin{cases}{[K(x)-E(x)],} & x \leq 1 \\ x[K(1 / x)-E(1 / x)], & x>1\end{cases}
$$

The response functions for finite $T$ are obtained simply by multiplying Eqs. (8) and (10-12) by the factor $\tanh (\omega / 4 T)$. The partial screening of the $A_{1 g}$ channel by long-range Coulomb forces comes from the observation that the square of the energy gap enters into the response function in Eq. (6). For the case of $d_{x^{2}-y^{2}}$ pairing symmetry, the energy gap squared contains a term which transforms according to $A_{1 g}$ symmetry which leads to a mixing of the $L=0$ and $L=4 A_{1 g}$ basis functions. This corresponds to partial "transverse screening" of the $A_{1 g}$ vertex [7].

The corresponding real parts were obtained via Kramers-Kronig analysis and are plotted together with the imaginary parts in Figure 1 for the $B_{1 g}$ and screened $A_{1 g}$ channels. We see that the peak in the imaginary part of the self-energy (which determines the linewidth of 
the phonon) lies at different frequencies $\omega_{\text {peak }} \sim 2 \Delta_{0}(T)$ and $1.2 \Delta_{0}(T)$ for the $B_{1 g}$ and $A_{1 g}$ channels, respectively. This is a consequence of the angular averaging which couples the gap and $e-p h$ vertex, and leads to constructive (destructive) interference under averaging if the vertex and the gap have the same (different) symmetry. Similar behavior for the electronic contribution to Raman scattering led to the reasoning that the symmetry which shows the highest peak position gives an unique indication of the predominant symmetry of the gap [7]. The symmetry dependence is also manifest in the low frequency behavior, which can be written as

$$
\begin{gathered}
\Sigma_{B_{1 g}}^{\prime \prime}(\omega \rightarrow 0)=3 N_{F} g_{B_{1 g}}^{2} x^{3} / 4+O\left(x^{5}\right), \\
\Sigma_{A_{1 g}}^{\prime \prime}(\omega \rightarrow 0)=N_{F} g_{A_{1 g}}^{2} x+O\left(x^{3}\right),
\end{gathered}
$$

i.e., the spectrum of the self energy rises slower in the $B_{1 g}$ channel than the $A_{1 g}$ channel [12]. The power laws are a signature of an energy gap which vanishes on lines on the Fermi surface, but the channel dependence of the exponents are unique to a $d_{x^{2}-y^{2}}$ pair state. These channel-dependent power-laws have been observed in the electronic contribution to Raman scattering in BSCCO, YBCO, and double and triple layer Thallium cuprates which constitute strong evidence for a $d$-wave gap of this symmetry as opposed to $d_{x y}$, $d_{x z}$ or $d_{y z}$ symmetry, which also have nodes on lines on the Fermi surface [7].

The real parts of the self energies (which determines the frequency renormalization) show a change of sign near the peak in the imaginary part. While the $B_{1 g}$ channel shows a mild frequency dependence away from the peak maximum and then a rapid change of sign at the

peak, the $A_{1 g}$ channel shows a smooth crossover from negative to positive values, with a change of sign that occurs at a frequency which is slightly greater than the peak maximum in the imaginary part. Thus a phonon of $A_{1 g}$ symmetry which lies at energies below $2 \Delta_{0}(T)$ can become hardened as opposed to softened. We immediately can draw the conclusion that phonons of the same frequency will show qualitatively different behavior in different channels as a consequence of their symmetry. Therefore, careful attention must be paid to symmetry before an analysis of the gap can be made by locating the point where phonon softening or hardening occurs.

\section{TEMPERATURE DEPENDENCE}

We now investigate the temperature dependence of the phonon lineshape. The $\mathbf{q}=0$ spectral function, Eq. (6), vanishes at $T_{c}$ due to the lack of particle-hole continuum for 
pair creation. This term thus always predicts phonon broadening compared to the normal state below $\mathrm{T}_{c}$ for a gap with nodes. However, the term responsible for the normal metal self energy (due to, eg., finite momentum transfer or anharmonic decay) will be affected by superconductivity due to the reorganization of the density of states as the gap opens up. In order to recover the normal metal lineshape at $T_{c}$, one must use finite q (or impurity scattering [10,13]) to generate the additional term $\delta \Sigma$ which does not vanish at $T_{c}$. We now generalize the result to finite $q$ for anisotropic gaps. For finite $q$, the spectrum of $\delta \Sigma$ at finite temperatures is given by $\delta \Sigma^{\prime \prime}(q, T, \omega)=\Theta\left(v_{F} q-\omega\right)\left\langle\left|g_{\hat{\mathbf{k}}}^{\gamma}\right|^{2} F(\hat{\mathbf{k}}, \omega)\right\rangle$ with

$$
\begin{aligned}
& F(\hat{\mathbf{k}}, \omega)=\frac{N_{F} \pi^{2}}{2 v_{F} q} \int_{|\Delta(\hat{\mathbf{k}})|}^{\infty} d E\left\{[f(E)-f(E+\omega)] \frac{E(E+\omega)-|\Delta(\hat{\mathbf{k}})|^{2}}{\sqrt{E^{2}-|\Delta(\hat{\mathbf{k}})|^{2}} \sqrt{(E+\omega)^{2}-|\Delta(\hat{\mathbf{k}})|^{2}}}\right. \\
& \left.\quad+\Theta(E-|\Delta(\hat{\mathbf{k}})|-\omega)[f(E-\omega)-f(E)] \frac{E(E-\omega)-|\Delta(\hat{\mathbf{k}})|^{2}}{\sqrt{E^{2}-|\Delta(\hat{\mathbf{k}})|^{2}} \sqrt{(E-\omega)^{2}-|\Delta(\hat{\mathbf{k}})|^{2}}}\right\},
\end{aligned}
$$

where $f$ is a Fermi function. The Theta function $\Theta\left(v_{F} q-\omega\right)$ restricts the frequency shift due to phase space consideration, reflecting that the region of the particle-hole continuum vanishes for small wavenumbers as a consequence of momentum conservation. Since $v_{F} q \ll \Delta$ in the cuprate materials and also in A-15 materials, this term will only contribute to the self energy for phonons of very small energy. However, it has been shown for $s$-wave superconductors [10] that the incorporation of impurity scattering removes the phase-space restriction due to the lifting of momentum conservation and $\delta \Sigma$ contributes for all frequencies. While incorporating impurity scattering remains beyond the scope of the present treatment, we remark that it is expected that a similar consideration for the case of $d$-wave superconductors would also lead to the contribution of $\delta \Sigma$ for all frequencies. This remains to be explored [14].

In the limit of small frequencies $(\omega<<T)$, we obtain the simple result

$$
\delta \Sigma^{\prime \prime}(q, \omega<<T)=\omega \frac{2 N_{F} \pi^{2}}{v_{F} q}\left\langle\frac{\left|g_{\hat{\mathbf{k}}}\right|^{2}}{e^{|\Delta(\hat{\mathbf{k}})| / T}+1}\right\rangle \Theta\left(v_{F} q-\omega\right) .
$$

Similarly, at $T_{c}$, Eq. (14) recovers $\delta \Sigma^{\prime \prime}\left(q, \omega<<T_{c}\right)=\Theta\left(v_{F} q-\omega\right) \omega \frac{N_{F} \pi^{2}}{v_{F} q}\left\langle\left|g_{\hat{\mathbf{k}}}^{\gamma}\right|^{2}\right\rangle$ and thus the ratio of the low frequency response in the superconducting state to that of a normal metal at $T_{c}$ is given by

$$
\frac{\delta \Sigma^{\prime \prime}(q, \omega<<T)}{\delta \Sigma^{\prime \prime}\left(q, \omega<<T_{c}\right)}=2 \frac{\left\langle\left|g_{\hat{\mathbf{k}}}^{\gamma}\right|^{2} f(|\Delta(\hat{\mathbf{k}})|)\right\rangle}{\left\langle\left|g_{\hat{\mathbf{k}}}^{\gamma}\right|^{2}\right\rangle} .
$$

This shows how the redistribution of the density of states below $T_{c}$ to higher energies as the gap opens up leads to a reduction of the decay channels available to particle-hole creation and 
a net decrease in the phonon linewidth. In isotropic superconductors, the Fermi function can be pulled out of the average and the resulting expression is independent of phonon symmetry. However, once the gap is anisotropic, there exists coupling between the vertex and the the gap which leads to a symmetry dependent result.

Using a weak coupling expression for the temperature dependence of the energy gap $\left(2 \Delta_{0} / k_{B} T_{c}=5.1252\right)$, we numerically evaluate Eq. (16) while taking screening into account. The results are plotted in Fig. 2 as a function of $T / T_{c}$ for a $d_{x^{2}-y^{2}}$ energy gap compared to a BCS isotropic gap. The low temperature behavior is given by a power-law in $T$ for all channels for the $d$-wave case while the ubiquitous exponential dependence in $T$ is seen for all channels in the $s$-wave case. The power-law behavior for the $d$-wave case is channel dependent, with exponents identical to those of Eq. (13), in the sense that $\omega$ can be replaced by $T$. What is remarkable is that the fall off of the Fermi function at low temperatures is quite slow in those channels which are orthogonal to the symmetry of the gap, with the notable example of the $A_{1 g}$ channel, which shows a residual broadening at $T / T_{c}=0.3$ of roughly 20 percent of that of the normal state. This was argued in the case of electronic Raman scattering to be further evidence for an energy gap in the cuprate materials which has predominantly $B_{1 g}$ character, due to the observation that a gap opens up quickly in the $B_{1 g}$ channel compared to $A_{1 g}$ and others which have been probed via Raman scattering [7].

For higher frequencies $\omega>T$, we have evaluated Eq. 14 directly. The results are quite similar to those of Fig. 2 for all frequencies $\omega$ up to roughly $4 \Delta$, but then at higher frequencies all channels eventually display a linear $T$ dependence (i.e., the behavior of the normal state) for energy scales much greater than the gap energy.

\section{ENTIRE SPECTRAL FUNCTION AND ROLE OF SATELLITES}

In this section we consider the entire phonon spectral function Eq. (1), paying particular attention to the role of satellites which arise due to $e-p h$ coupling. The role of satellites have not been explored in anisotropic superconductors. As is well known for BCS superconductors, satellites appear in the phonon spectral function for all frequencies of the optical phonon, but have the greatest residue for phonons near twice the gap edge. In the BCS case, impurities wipe out the satellite peak [10], explaining why they have yet to be definitively observed in conventional A-15 superconductors. In the absence of impurities however vastly different lineshapes can be obtained due to the interference of the satellites.

Using a gap of $d_{x^{2}-y^{2}}$ symmetry and working specifically at $q=0$, Eq. (6), we find 
that the satellites are present in anisotropic superconductors as well due to the fact that the real part of the denominator of Eq. (1) has two zeroes for any value of $\omega_{0}$ - one at the renormalized phonon frequency and the other at the satellite position. The satellite becomes more pronounced the closer the optical phonon is to the peak position of the self energy spectrum as in the BCS case and interferes with the phonon. Therefore, for the case of a phonon located below the spectral maximum, where the satellite peak is observable only at $\mathrm{T}=0$ for large $e-p h$ coupling, as the gap decreases on approaching $\mathrm{T}_{c}$ the satellite will be made to pass through the phonon position and will be subsequently distorted. This is shown in Fig. 3 for a $B_{1 g}$ phonon $\left(\omega_{0} / \Delta_{0}(T=0)=1.0, g_{B_{1 g}}^{2} N_{F} / \Delta_{0}(T=0)=0.1\right)$ for the temperatures indicated. The phonon lineshape is drastically affected by the satellite which takes spectral weight away from the phonon when the peak of the spectral function is close to the phonon position. The phonon linewidth grows as the peak of the spectrum moves up in energy with decreasing $T$ and is hardened. The linewidth and frequency shift reaches a maximum when the peak and phonon position coincide and then the linewidth decreases and the phonon softens as $T \rightarrow T_{c}$.

\section{CONCLUSIONS AND COMPARISON WITH EXPERIMENT}

In this Section we combine the previous results and examine the phonon linewidth as a function of temperature for the case of two phonons which lie at approximately 285,340 $\mathrm{cm}^{-1}$ for the $B_{1 g}$ channel and $464,500 \mathrm{~cm}^{-1}$ for the $A_{1 g}$ channel in BSCCO and YBCO, respectively. Using our previous fits to the electronic Raman scattering in BSCCO, we obtained a value of the energy gap at $T=0$ to be $\Delta_{0}(T=0)=287 \mathrm{~cm}^{-1}$ [7]. Therefore the normalized optical phonon frequency is given by $\omega_{0} / \Delta_{0}(T=0) \sim 0.99,1.62$ for the $B_{1 g}, A_{1 g}$ phonon, respectively in BSCCO, while for YBCO the ratio is expected to be slightly higher. We can immediately make the following statement. Since the interference effects of the phonon with the satellite peak can only occur for a phonon which is located at $T=0$ below the peak in the imaginary part of the self energy, there should be no interference effects on the $A_{1 g}$ phonon since it lies above the peak in the spectrum at $T=0$. Thus its renormalization should be a monotonic function of temperature. However that is not the case for the $B_{1 g}$ phonon. Anomalous behavior of the $B_{1 g}$ renormalization as seen in Fig. 3 arises due to the interference between the phonon and the rapid rise of the self energy near $2 \Delta_{0}(T)$, which passes through the phonon frequency at $\mathrm{T} / \mathrm{T}_{c} \sim 0.9$. Another remark is in order. In order to make an accurate fit to the data, the magnitude of the coupling 
constant needs to be addressed. As we have seen in Section IV, it controls the strength of the satellite and its subsequent effect on the phonon lineshape. Little is known about the coupling constant [8] and thus we can only make general statements on the behavior of the phonons. The magnitude of the effect cannot be predicted.

Inspecting Fig. 1, the $\mathbf{q}=0$ part of the self energy, at each phonon frequency, we see that the $B_{1 g}$ phonon is broadened and softened at $T=0$ compared to $T_{c}$ while the $A_{1 g}$ phonon is broadened but lies right at the point where the real part is changing sign. This term most accurately describes what is seen in the phonons in YBCO. A rapid rise of the $B_{1 g}$ phonon linewidth below $T_{c}$ has been seen [6.15], reflecting the interference of the peak in the self energy and the phonon, (see Eq. (6) and Fig. 3). The $B_{1 g}$ phonon additionally is mostly softened to lower frequencies. For the $A_{1 g}$ phonon, the frequency shift is seen only to slightly higher frequencies. This is due to the real part of the self energy crossing zero at the phonon position. However, the $A_{1 g}$ phonon narrows just below $T_{c}$, reaches a minimum width and then broadens at lower temperatures. This could be a result of a competition between the effects of $\Sigma$ and $\delta \Sigma$, but without information concerning the magnitude of the coupling constant, finite momentum transfer or impurity scattering this remains an open question.

In addition, we have the contribution arising from non-zero $q$, Eq. (14), which indicates that this contribution to the self energy is reduced compared to its value at $T_{c}$ and decreases as $T^{3}$ and $T$ for low temperatures in the $B_{1 g}$ and $A_{1 g}$ channels, respectively (see Fig. 2). This term most accurately describes the experiments in BSCCO. The linewidth of both the $B_{1 g}$ and $A_{1 g}$ phonons decrease monotonically with temperature, which points to the lack of contribution coming from satellite effects. The linewidth decrease in the $B_{1 g}$ channel can be fit with a $T^{3}$ dependence while a term linear in $T$ can be fit to the $A_{1 g}$ phonon which is the same dependence as in the normal state. Both behaviors are consistent with Eq. (16). This is to be compared with the predictions of an isotropic $s$-wave theory lines, which are identical for each channel $\left(\sim e^{-\Delta / T}\right)$. The theory for a gap of $B_{1 g}$ character shows a marked symmetry dependence resulting from the interplay of gap and vertex symmetry. While it is arguable whether an exponential temperature dependence can also fit the $B_{1 g}$ data [5], the lack of change of the exponent of the $A_{1 g}$ phonon's linewidth is a direct consequence of the energy gap anisotropy. Therefore it will appear that the $A_{1 g}$ phonon will be unaffected by superconductivity. Since the $B_{1 g}$ phonon has the same symmetry of a $d_{x^{2}-y^{2}}$ gap, it's linewidth will show the greatest change due to the onset of superconductivity.

In conclusion, within the accuracy of the experiments, we have seen that the changes 
in the phonon lineshape as a function of symmetry can be explained with a choice of the gap which has (at least predominantly) $B_{1 g}$ character, supporting recent comparisons made on electronic Raman scattering on BSCCO. However, without knowledge of the magnitude of the coupling constant, the importance of finite $q$ and satellite effects remains an open question. Of course other choices of gaps which have a small but finite minimum value, eg. anisotropic $s$-wave or $s+i d$ would give similar results to the $d_{x^{2}-y^{2}}$ choice for the gap. Both the electronic and phonon contributions to Raman scattering below $T_{c}$ can be explained by simply invoking the symmetry of the vertex which couples to the symmetry of the gap. More detailed experiments would be extremely useful to pin down the magnitude of the $e-p h$ vertex and subsequently the role of the satellites, and the role of impurities and the mechanism and magnitude of the coupling remains to be explored [8,14].

\section{ACKNOWLEDGMENTS}

I am indebted to Drs. D. Einzel, A. Virosztek and A. Zawadowski for many valuable discussions and a critical reading of the manuscript. I would also like to thank D. Leach for discussions and for providing me with the data discussed in the text prior to publication. Similarly, I would also like to acknowledge helpful suggestions and discussions from Drs. R. Hackl, E. Nicol, B. Stadlober, and G. Zimanyi. I would like to acknowledge the hospitality of A. Virosztek and A. Zawadowski and their colleagues at the Research Institute for Solid State Physics of the Hungarian Academy of Science and the Institute of Physics of the Technical University of Budapest, where part of this work was completed. This work was supported by N.S.F. grant 92-06023 and the American Hungarian Joint Grant number NSF 265/92b. 


\section{REFERENCES}

1 C. Thomsen and M. Cardona, in Physical Properties of High $T_{c}$ Superconductors, Vol. I, edited by D. M. Ginsberg (World Scientific, Singapore, 1989).

$2 \quad$ R. Zeyher and G. Zwicknagl, Z. Phys. B 78, 175 (1990).

3 E. J. Nicol, C. Jiang, and J. P. Carbotte, Phys. Rev. B 47, 8131 (1993).

4 G. Burns, G. V. Chandrashekhar, F. H. Dacol, and P. Stroebel, Phys. Rev. B 39, 775 (1989); M. Boekholt, A. Erle, P. C. Splittgerber-Hünnekes, and G. Güntherodt, Solid State Comm. 74, 1107 (1990).

5 D. H. Leach, C. Thomsen, M. Cardona, L. Mihaly, and C. Kendziora, Solid State Comm. 88, 457 (1993).

6 E. Altendorf, X. K. Chen, J. C. Irwin, R. Liang and W. N. Hardy, Phys. Rev. B 47, 8140 (1993), and references therein.

7 T. P. Devereaux, D. Einzel, B. Stadlober, R. Hackl, D. H. Leach and J. J. Neumeier, Phys. Rev. Lett. 72, 396 (1994); T. P. Devereaux, D. Einzel, B. Stadlober and R. Hackl, ibid. 72, 3290 (1994).

$8 \quad$ T. P. Devereaux, A. Virosztek and A. Zawadowski, unpublished.

$9 \quad$ T. P. Devereaux and D. Einzel, preprint.

10 T. P. Devereaux, Phys. Rev. B 45, 12965 (1992); ibid. 47, 5230 (1993).

11 M.V. Klein and S. B. Dierker, Phys. Rev. B 29, 4976 (1984); H. Monein and A. Zawadowski, Phys. Rev. B 41, 8798 (1990).

12 We note that the logarithmic singularity in the $B_{1 g}$ channel is due to the two dimensionality of the Fermi surface. Adding $z$ dispersion removes the singularity and replaces it with a cusp, as seen in Ref. [7]. The peak position is unchanged in the $B_{1 g}$ channel but the low frequency behavior is modified by a $\log \left(\left|2 \Delta_{0} / \omega\right|\right)$ prefactor to the $\omega^{3}$ behavior. For the $A_{1 g}$ channel, the presence of $z$ dispersion allows the $L=2$ channel to contribute. The $L=2 A_{1 g}$ channel has a peak at lower frequencies than the $L=4$ channel, $\left(\omega_{\text {peak }} \sim 0.6 \Delta_{0}\right)$, and also has the same log prefactor as above to the linear $\omega$ dependence, turning the low frequency behavior into a convex shape [7]. Therefore, for a more realistic Fermi surface applicable to the cuprates which is predominantly 2-D 
in nature, the logarithmic singularity in the $B_{1 g}$ channel can be cut off while the $A_{1 g}$ channel can become more convex in shape and have a peak resulting from the superposition of the $L=2$ and $L=4$ contributions which is slightly lower than that of a pure 2-D Fermi surface.

13 A. Zawadowski and M. Cardona, Phys. Rev. B 42, 10732 (1990).

14 T. P. Devereaux, unpublished.

15 S. L. Cooper, F. Slakey, M. V. Klein, J. P. Rice, E. D. Bukowski, and D. M. Ginsberg, Phys. Rev. B 38, 11934 (1988).

16 E. Altendorf, J. Chrzanowski, J. C. Irwin and J. Franck, Solid State Comm. 76, 391 (1990). 


\section{FIGURES}

FIG. 1. Real and imaginary parts of the phonon self energy for the $B_{1 g}$ and $A_{1 g}$ channels for a cylindrical Fermi surface. Magnitude of the vertices are set equal to one.

FIG. 2. Temperature dependence of the $\omega \rightarrow 0$ imaginary part of the self energy in a $d_{x^{2}-y^{2}}$ and isotropic BCS superconductor compared to the normal state.

FIG. 3. Phonon spectral function for a $B_{1 g},\left(\omega_{0}=\Delta_{0}(T=0), g_{B_{1 g}}^{2} N_{F} / \Delta_{0}(T=0)=0.1\right)$, phonon in a superconductor with $d_{x^{2}-y^{2}}$ pairing symmetry for various values of $T / T_{c}$ as indicated in upper part of Figure. 